\title{
Genetic interactions between Protein Kinase $D$ and Lobe mutants during eye development of Drosophila melanogaster
}

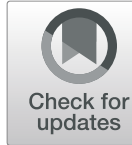

\author{
Dieter Maier ${ }^{\dagger}$, Anja C. Nagel ${ }^{\dagger}$ and Anette Preiss ${ }^{*}$ (D)
}

\begin{abstract}
Background: In Drosophila, the development of the fly eye involves the activity of several, interconnected pathways that first define the presumptive eye field within the eye anlagen, followed by establishment of the dorso-ventral boundary, and the regulation of growth and apoptosis. In Lobe (L) mutant flies, parts of the eye or even the complete eye are absent because the eye field has not been properly defined. Manifold genetic interactions indicate that $L$ influences the activity of several signalling pathways, resulting in a conversion of eye tissue into epidermis, and in the induction of apoptosis. As information on the molecular nature of the $L$ mutation is lacking, the underlying molecular mechanisms are still an enigma.
\end{abstract}

Results: We have identified Protein Kinase D (PKD) as a strong modifier of the $L$ mutant phenotype. PKD belongs to the PKC/CAMK class of Ser/Thr kinases that have been involved in diverse cellular processes including stress resistance and growth. Despite the many roles of PKD, Drosophila PKD null mutants are without apparent phenotype apart from sensitivity to oxidative stress. Here we report an involvement of PKD in eye development in the sensitized genetic background of Lobe. Absence of PKD strongly enhanced the dominant eye defects of heterozygous $L^{2}$ flies, and decreased their viability. Moreover, eye-specific overexpression of an activated isoform of PKD considerably ameliorated the dominant $L^{2}$ phenotype. This genetic interaction was not allele specific but similarly seen with three additional, weaker $L$ alleles $\left(L^{1}, L^{5}, L^{G}\right)$, demonstrating its specificity.

Conclusions: We propose that PKD-mediated phosphorylation is involved in underlying processes causing the $L$ phenotype, i.e. in the regulation of growth, the epidermal transformation of eye tissue and apoptosis, respectively.

Keywords: Drosophila melanogaster, Protein kinase D, PKD null mutant, Lobe, Eye development

\section{Background}

The Drosophila eye develops from the eye-antennal imaginal disc that eventually gives rise to the eye, the antenna and large parts of the head capsule (overview in: [1-3]). The master control genes for eye development in all seeing animals studied to date are encoded by the Paired box 6 (Pax6) gene family (overview in: [3-5]). The founding members in D. melanogaster, eyeless (ey) and twin of eyeless (toy), are at the top of a network of retinal determination genes required for growth and the specification of the presumptive retinal field within the eye anlagen. One major task thereby is the suppression

\footnotetext{
* Correspondence: a.preiss@uni-hohenheim.de

${ }^{\dagger}$ Dieter Maier and Anja C. Nagel contributed equally to this work.

Universität Hohenheim, Institut für Genetik (240A), Garbenstr. 30, 70599 Stuttgart, Germany
}

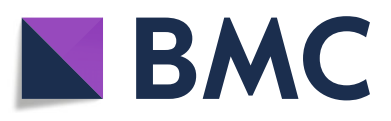

(c) The Author(s). 2019 Open Access This article is distributed under the terms of the Creative Commons Attribution 4.0 International License (http://creativecommons.org/licenses/by/4.0/), which permits unrestricted use, distribution, and reproduction in any medium, provided you give appropriate credit to the original author(s) and the source, provide a link to the Creative Commons license, and indicate if changes were made. The Creative Commons Public Domain Dedication waiver (http://creativecommons.org/publicdomain/zero/1.0/) applies to the data made available in this article, unless otherwise stated.

of non-ocular selector genes within the eye field, which otherwise direct the formation of head epidermis (overview in: [3]). Accordingly, retinal identity is lost in ey mutants accompanied by massive cell death, and ey; toy double mutants are even headless [6-10] (overview in: [3]). Several signalling pathways coordinate eye development, including the Wingless $(\mathrm{Wg})$ and Notch $(\mathrm{N})$ pathways. Whereas $\mathrm{Wg}$ is important for confining the presumptive eye field within the eye anlagen, $\mathrm{N}$ activity is required for establishing the dorso-ventral boundary as the major growth center (Fig. 1) (overview in: [2, 3]). Early on, the eye primordium is of ventral fate; formation of the dorsal compartment requires Wg signalling activity, which eventually triggers the activation of $\mathrm{N}$ along the midline (Fig. 1) [11-15]. 

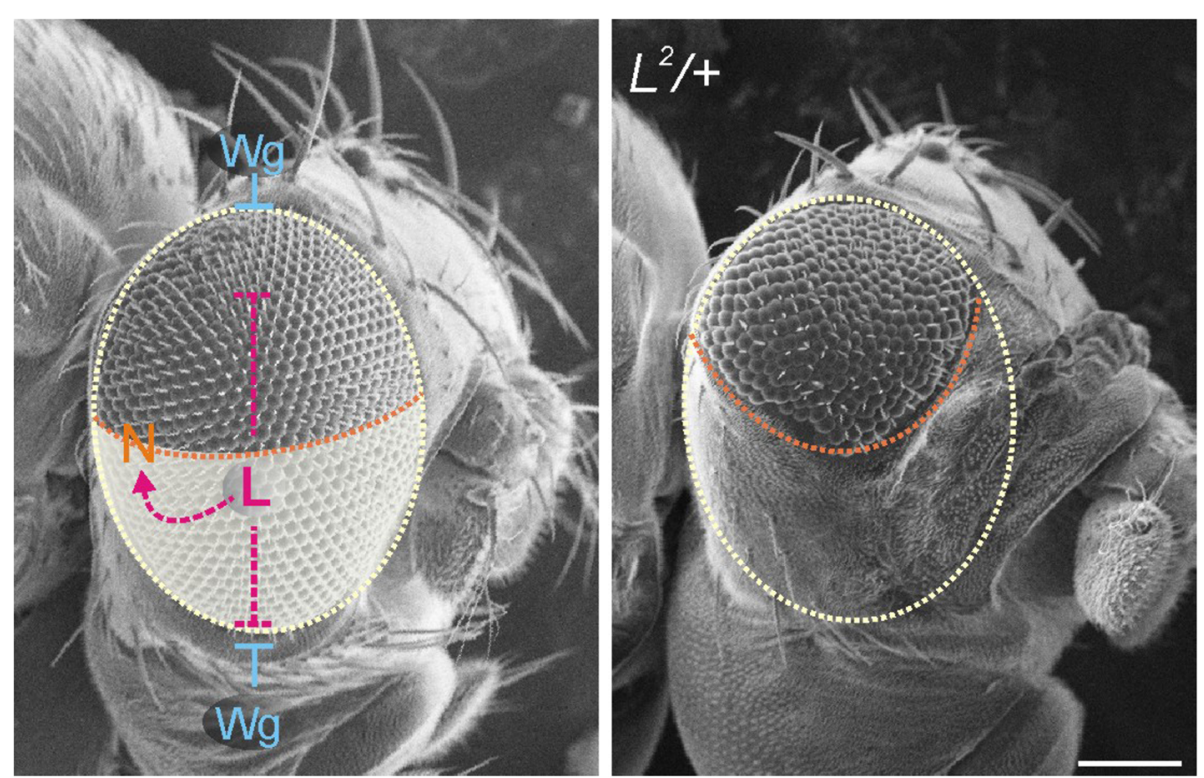

Fig. 1 Development of the ventral eye is affected by the $L$ mutation. During development, the Drosophila eye field is confined by the activity of Wg. Establishment of the dorso-ventral boundary requires the activation of N. Concluded from genetic interactions, Lobe acts as a positive regulator of $\mathrm{N}$ and a negative regulator of $\mathrm{Wg}$ activities. Accordingly, $L^{2} /+$ mutant flies display small eyes, where the ventral eye compartment is reduced at the expense of head epidermis. Arrows indicate positive, and bars negative interactions. Size bar, $100 \mu \mathrm{m}$

In Lobe ( $L$ ) mutant flies, eye development is disturbed: notably the ventral eye field is affected and in extreme cases, the complete eye is lacking (Fig. 1) [16]. Generally, the phenotypic character is very variable, and $L$ mutant flies typically are strongly asymmetric. Whereas most $L$ alleles overlap wild type in heterozygosis, and display reduced eyes only in homozygosis, the most extreme allele $L^{2}$ is fully dominant and of poor viability in homozygosis [16]. Genetically, $L^{2}$ is a neomorphic, dominant mutation since the mutant eye phenotype is reverted by deletions. Revertants are homozygous lethal at embryonic stage, but the corresponding gene has not been identified so far [17, 18]. $L$ defects can be traced to the early development of the eye precursor: Fewer cells enter into eye disc formation, and the cephalic complex is significantly reduced in size already in first instar larva [16, 18-21]. $L$ has been tightly linked to $\mathrm{Wg}$ and $\mathrm{N}$ signalling pathways in opposing ways $[18,22,23]$. With regard to $\mathrm{N}$ activity, $L$ acts as a positive factor, being involved in the regulation of growth and survival of cells in the ventral compartment $[18,23]$. In contrast, $\mathrm{Wg}$ and $L$ act antagonistically. For example, the $L$ mutant eye phenotype is enhanced by increased $\mathrm{Wg}$ activity, whereas it is ameliorated by $w g$ mutants or by the overexpression of the Ser/Thr kinase Shaggy (Sgg), a known antagonist of Wg signalling [23]. In fact Wg is upregulated in ventral eye tissue mutant for $L$, resulting in a conversion of eye tissue into epidermis and induction of apoptosis (Fig. 1) [23-25].

Interestingly, the $L$ mutant phenotype is exquisitely sensitive to genetic background [16, 21, 22]. For example, it is enhanced in a Minute background like the M (3)95A mutation, i.e. by the general downregulation of protein synthesis through mutation of ribosomal proteins like RpS3 [26, 27], suggesting a role for $L$ in growth regulation. The proposed link between $L$ and the negative TOR- regulator Pras40, however, has not been confirmed [28-30]. To date the molecular basis of the $L$ mutation is still unknown. Hence, the molecular mechanisms underlying the $L$ phenotype remain an enigma.

Here we show that Protein Kinase D (PKD) is a strong modifier of the $L$ mutant phenotype. PKD is a member of the PKC/CAMK family of Ser/Thr kinases, and has been involved in diverse cellular processes including growth regulation, protection from oxidative stress, Golgi-mediated protein transport, as well as regulation of actin cytoskeletal dynamics in mammals (reviewed in: [31-34]), as well as in Drosophila [35-39]. Despite these many roles, Drosophila PKD null mutants are without apparent external phenotypes [40]. Moreover, mutant flies are not significantly different from control regarding fertility, longevity, growth and resistance to a variety of stressors including starvation apart from sensitivity to oxidative stress [40]. Apparently, PKD acts redundantly with other kinase members of the PKC/CAMK family, including PKC $\delta$, Sqa and Drak [40]. In the course of the genetic combination of the $P K D$ mutant with kinase candidates, we noted an unexpected, strong genetic interaction between the null allele $P K D^{26}$ and $L^{2}$ : the dominant mutant eye phenotype was strongly enhanced, and viability of $L^{2}$ heterozygotes markedly decreased. 
Specificity of the genetic interaction was confirmed by a rescue of the $L^{2}$ eye phenotype through eye-specific overexpression of the activated isoform of PKD, PKDSE. Finally, we show that this interaction is not allele specific but similarly seen with three additional, weaker $L$ alleles $\left(L^{1}, L^{5}, L^{G}\right)$. We propose that PKD-mediated phosphorylation is involved in the molecular mechanisms underlying the aberrant eye development in $L$ mutants.

\section{Results}

The small eye phenotype of $L^{2}$ is controlled by protein kinase $D$ activity

In our conditions, the vast majority of $L^{2}$ heterozygotes displayed an intermediate phenotype where both eyes are smaller (representative examples are shown in Fig. 1 and Fig. 2a, a'). In a PKD null mutant genetic background, i.e. combined with homozygous $P K D^{26}$, the phenotype was strongly enhanced, since most flies displayed one or both eyes of pinhead size or complete absence (Fig. 2a', b'). To account for the high phenotypic variability between the two eyes, and to allow a quantification of the genetic influence of $P K D$ on $L$, we classified the phenotypes into five groups. $L^{2}$ heterozygotes predominantly fell in class 2 (c2) (Fig. 2b', d). Yet, in the absence of $P K D$ nearly $90 \%$ of the flies were grouped into class 3 or 4 (c3, c4) respectively, i.e. no or little leftovers of one or both eyes (Fig. 2b', d), in agreement with a strong requirement of PKD activity during $L$-dependent eye development.

It is well known that the $L$ phenotype is exquisitely sensitive to environmental and genetic background [16, $21,22]$. As the $P K D^{26}$ allele was generated by homologous recombination [40], the parental background cannot be easily reconstituted. Hence, other modifiers elsewhere on the third chromosome might be responsible for the phenotypic enhancement of $L^{2}$ [21]. If, however, $P K D$ were the culprit, overexpression of an activated form of the kinase would be expected to cause the opposite result, i.e. a rescue of the eye defects. Accordingly, the wild type PKD isoform should have little influence, as PKD activity requires its phosphorylation by upstream activating kinases [32], whereas a dominant negative isoform may enhance the $L^{2}$ mutant eye phenotype. To confirm the specific role of PKD, we overexpressed the activated PKD-SE form specifically in the developing eye tissue, using the Gal4/UAS-system [36, 41]. To this end, $L^{2}$ was combined with either ey-Gal4 [42], or with UAS-PKD-SE [36]. Whereas overexpression of UAS-PKD-SE in the eye anlagen does not alter the eye morphology (Fig. 2c), the offspring resulting from the subsequent cross indeed displayed a much milder phenotype: the eyes were generally larger (class1, kidney shaped, instead of class2, halved) (Fig. 2a', c'), and frequently, only one of the two eyes was affected. The apparent impression was confirmed by a quantification of the results: a third of the flies was clearly rescued compared to their siblings (Fig. 2d). Similarly, UASPKD-WT and UAS-PKD-kd, encoding a wild type and presumptive 'kinase dead' PKD isoform, respectively [36], were overexpressed in the eye anlagen of $L^{2}$ mutant larvae, and the resultant phenotypes were quantified (Fig. 2d). Compared to the control $L^{2} /+$; ey-Gal4/+ flies, overexpression of UAS-PKD-WT had little impact on eye development, whereas UAS-PKD-kd caused a slight enhancement, as predicted (Fig. 2d). These results clearly demonstrate the strong and specific influence of PKD activity on eye development in the $L^{2}$ mutant background.

\section{Viability of $L^{2}$ is impaired by the absence of $P K D$}

In the course of the above experiments we noted that the number of $L^{2}$ heterozygous flies lacking PKD (i.e. $L^{2} /$ +; $\left.P K D^{26} / P K D^{26}\right)$ was considerably lower compared to the siblings. The aberrancy was determined by quantifying the offspring from a cross of $L^{2} / C y O ; P K D^{26} / \mathrm{TM} 3$ $S b$ males with virgins $P K D^{26} / P K D^{26}$. Four equal fractions with either homo- or heterozygous $P K D^{26}$ genotype were expected. The homozygous $P K D^{26}$ fraction carrying the $L^{2}$ allele in one copy, however, reached only $12 \%$ of the expectation (Fig. 3). Apparently, viability of flies lacking PKD function is strongly impaired in the presence of the $L^{2}$ allele.

\section{Genetic interactions between PKD and Lobe are not allele specific}

$L^{2}$ is the most extreme Lobe allele available with a fully penetrant dominant eye phenotype, and strongly reduced viability in the homozygotes. Other $L$ alleles are generally weaker, and the heterozygous phenotype overlaps wild type [16]. In homozygosis, the alleles $L^{1}, L^{5}$ and $L^{G}$ are viable and display variably strong eye defects (Fig. 4a-d) [16]. In our hands, $L^{1}$ homozygotes displayed a fully penetrant phenotype, with the majority of flies developing strongly reduced eyes (Fig. $4 \mathrm{~b}$, e). The two other alleles were weaker: about $20 \%$ of the $L^{5}$ and more than $60 \%$ of the $L^{G}$ homozygotes overlapped wild type at $25^{\circ} \mathrm{C}$ (Fig. 4c-e); eye defects were generally restricted to kidney shaped incisions (Fig. 4c, d). As the $L$ phenotype is highly variable, it is difficult to generate a robust phenotypic series [16]. In the $P K D^{26}$ homozygous background, however, the phenotypes were considerably enhanced; up to $30 \%$ of the flies now displayed only pinhead eye size, or lacked one or both eyes altogether (Fig. 4a'-d', e). Interestingly, absence of PKD had the most obvious impact on the weakest allele $L^{G}$, and the doubly homozygotes displayed as strong phenotypes as the $L^{1}$; $P K D^{26}$ combination (Fig. 4b', d', e). 


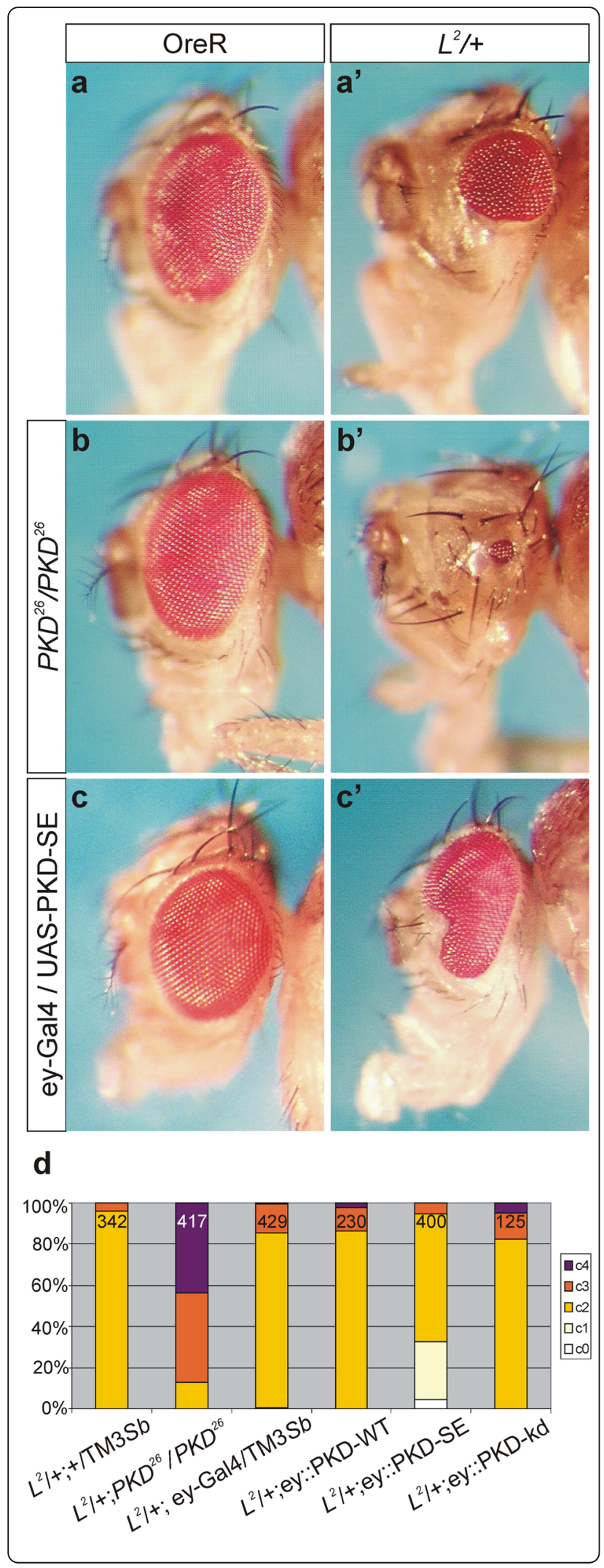

Fig. 2 Genetic interaction of PKD with $L^{2}$. Head of female flies; the left column shows a wild type OreR background (a), the right column a $L^{2} /+$ heterozygous background ( $\left.a^{\prime}\right)$. PKD ${ }^{26}$ homozygotes have wild type eyes (b). Yet, lack of PKD strongly enhances the dominant $L^{2}$ small eye phenotype $\left(b^{\prime}\right)$. Overexpression of UAS-PKDSE in the developing eye with ey-Gal4 is itself without apparent phenotype (c), but rescues the small eye phenotype of $L^{2} /+$ mutant flies $\left(c^{\prime}\right)$. (d) The dominant $L^{2}$ eye phenotype is backgrounddependent and often varies between both eyes. For a quantitative analysis of the genetic interaction, the $L^{2}$ eye phenotype was classified as follows: $\mathrm{c0}$, wild type eyes; $\mathrm{c1}$, one eye is smaller; $\mathrm{C2}$, both eyes are smaller; $c 3$, one eye is smaller and one is of pinhead size or absent; $c 4$ both eyes are of pinhead size or absent.

Genotypes are depicted below each column. The number of flies of the given genotype assessed in the experiment is indicated within each column. Note the strong enhancement of the $L^{2} /+$ phenotype by the absence of PKD and its rescue by overexpression of PKD-SE. Overexpression of PKD-WT had no impact, and that of PKD-kd caused little enhancement

We again assessed the effect of the activated form of PKD on the extent of the $L$ small eye phenotype in the background of the three different $L$ alleles (Fig. 5). To this end, each $L$ allele was combined with either UASPKD-SE or with ey-Gal4. The resultant flies were crossed, and their offspring analysed for eye defects (Fig. $\left.5 \mathrm{a}-\mathrm{d}^{\prime}\right)$. As observed for $L^{2}$, PKD-SE markedly rescued the recessive eye phenotype of either $L^{1}, L^{5}$ or $L^{G}$ (Fig. 5 e), confirming the specificity of the genetic interaction between the two genes. Again, $L^{G}$ appeared most susceptible to the influence of PKD, since nearly half of the flies were phenotypically wild type (Fig. 5d', e).

\section{Discussion}

Our work identified striking genetic interactions between $P K D^{26}$ and Lobe mutants, as the small eye phenotype of several $L$ alleles was strongly enhanced in the absence of $P K D$, and ameliorated by activated PKD-SE overexpressed within the eye anlagen. Moreover, lethality of $L^{2}$ heterozygotes was markedly increased in the $P K D^{26}$ mutant background. Overall, our data reveal a requirement of PKD activity for eye development and fly survival, uncovered in the sensitized genetic background of Lobe. Despite the fact that PKD has been involved in rhodopsin Rh1 homeostasis in the adult retina, eye morphogenesis is unaffected in the mutants [36, 43]. Being a member of PKC/CAMK family of Ser/Thr kinases kinases, PKD most likely regulates both aspects of development, eye development and fly viability, by specific protein phosphorylation. Interestingly, the genetic link between $L$ and several signalling pathways frequently involves Ser/Thr kinases or components that are phosphotargets thereof. Examples for respective kinases are hemipterous, a component of MAPK and JNK signalling, and shaggy, a negative regulator of $\mathrm{Wg}$ signalling activity, that modify the $L$ phenotype when mutant $[22,23] . L$ 


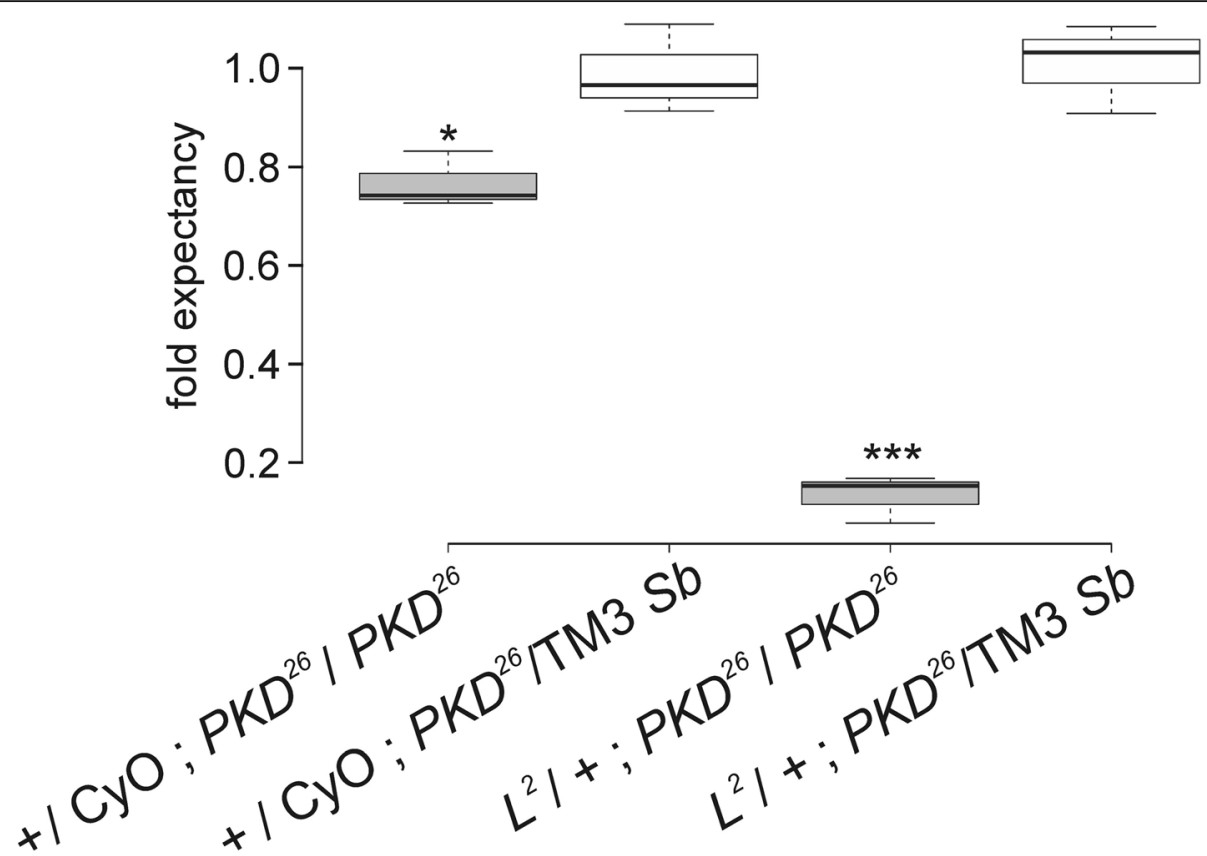

Fig. 3 Viability of $L^{2}$ heterozygotes is lowered in the absence of $P K D$. Viability of $P K D^{26}$ homozygotes (labelled grey) in a $L^{2} /+$ heterozygous background, compared to the doubly heterozygous siblings derived from a cross of $L^{2} / C y O ; P K D^{26} / T M 3 S 6$ males with virgins $P K D^{26} / P K D^{26}$. Each genotype is expected with the same frequency; the $P K D^{26}$ homozygotes however, reach only about $12 \%$ expectancy in the background of $L^{2}$ (0.12). Center lines of BoxPlots display the median, box limits indicate the 25th and 75th percentiles as determined by R-software, whiskers extend 1.5 times the interquartile range. Three experiments were performed with 333 total number of flies. Statistical significance of probes was determined by ANOVA two-tailed test for multiple comparisons using Dunnett's approach relative to the doubly heterozygous control, with raw $p$-values: $p>0.05$ (not significant); ${ }^{*} p<0.05 ;{ }^{* *} p<0.01 ;{ }^{* * *} p<0.001$

interactors that are themselves phospho-targets are involved in MAPK-, JNK-, Wg-, Hh-, Dpp-, N-, and Jak/ Stat-signalling pathways $[22,23]$, i.e. in the regulation of cell growth and apoptosis, and ultimately in eye development. Most likely PKD feeds into these pathways either directly or by shared phospho-targets.

A striking feature of the $L$ mutant phenotype is the asymmetry, i.e. one eye may be lacking whereas the other may be nearly normal $[16,19,21]$. Similar defects are found in flies mutant for eyeless (ey) [6, 16, 44-47], the master regulator of eye development encoding the Drosophila Pax6 homologue (overview in: [4, 5, 48]). The major role of ey is to control eye specification, and the survival and proliferation of eye progenitor cells [10, 45-47]. Similar to $L$, the ey mutant phenotype results from massive cell death within the eye anlagen of young larvae [7, 8, 45]. In fact, inhibition of apoptosis largely rescued lethality of $e y^{D}$ mutants, resulting in completely eyeless adults [9]. Loss of the ventral eye field in $L$ is likewise rescued by increased levels of cell death inhibitors or a downregulation of pro-apoptotic genes, indicating that apoptosis is a predominant trigger for the $L$ eye defects [23]. In vertebrates, the requirement for PKD in the regulation of apoptosis is well established [32, 49]. Perhaps $P K D$, in concert with $L$, normally acts as a survival signal during early eye development. Normal flies are strikingly symmetrical with little size-differences between left and right, despite the fact that the two body parts and their appendages grow independently as imaginal discs to be fused only during pupal development [1]. This is also true for the two wild type eyes that show only small differences in the number of their ommatidia [50]. The Drosophila insulin like peptide Dilp8 mediates the homeostatic regulation through the coordination of growth of imaginal discs. It triggers a neurosecretory circuit by activating its receptor Lgr3 (Leucine-rich repeat containing $G$ protein-coupled receptor 3) in a pair of neurons in the brain that act on downstream neuroendocrine cells. Eventually, growth of imaginal tissues is synchronized to maintain size proportions, thereby ensuring bilateral symmetry [51-55]. The striking asymmetry characterizing both $L$ and ey mutants indicates a failure of coordinate growth regulation. Both, $L$ and $e y$ contribute to cell proliferation in the eye anlagen [10, 18, 23]. Moreover, ey controls the differentiation and function of insulin-producing cells within the larval brain, and thereby systemic growth of the whole animal [56]. Albeit a link to the Dilp8-mediated neurosecretory circuit has not yet been established, it clearly must integrate organ growth and systemic growth coordination $[55,57]$. We speculate that it may also involve the activity of PKD. 

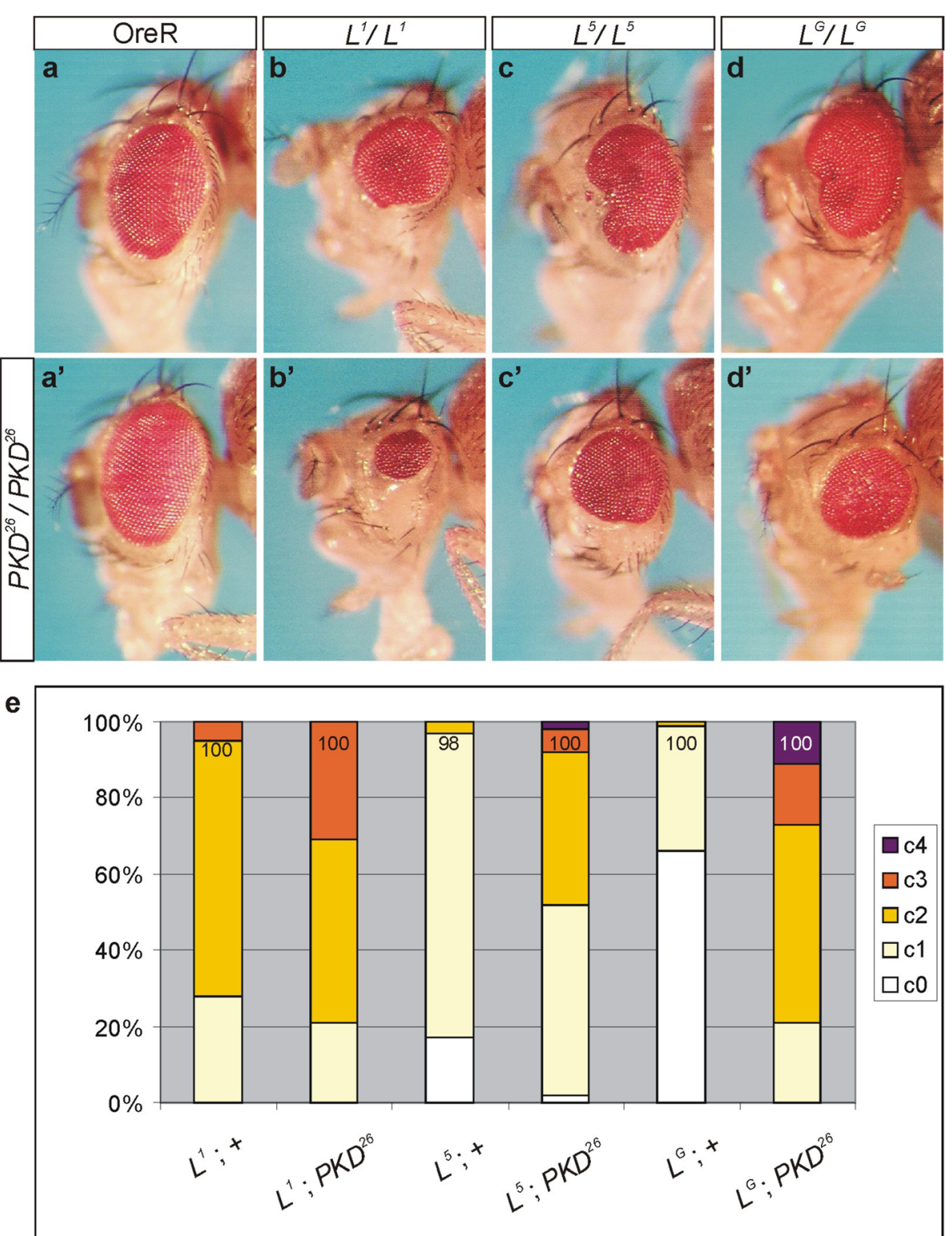

Fig. 4 Phenotypic enhancement of recessive $L$ alleles by the absence of PKD. Typical examples of female heads are shown. The upper row represents OreR as control (a) and three homozygous Lobe alleles, $L^{7}(\mathbf{b}), L^{5}(\mathbf{c})$ and $L^{G}(\mathbf{d})$. The lower row shows a typical example of a $P K D^{26}$ homozygous mutant female ( $\left.a^{\prime}\right)$ in the above genetic background $\left(b^{\prime}\right.$-d'): note enhancement of the $L$ small eye phenotype in the absence of PKD, independent of the $L$ allele. (e) Quantification of eye phenotypes in the given genotype by using the following classification: $\mathrm{cO}$, wild type eyes;

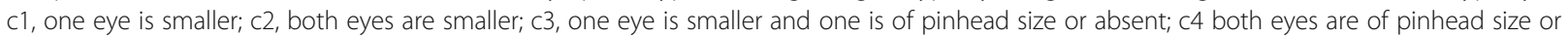
absent. Genotypes are depicted below each column; (+) indicates third chromosome normal for PKD. Crosses were performed at $25^{\circ} \mathrm{C}$. Fractions of phenotypes are depicted; numbers of assessed females are indicated in each column

Our recent work shows that $P K D$ is dispensable for normal fly development, however, presumably acts redundantly with other members of the PKC/CAMK family. Three kinases, Pkc $\delta$, Drak, and Sqa were uncovered as candidates for functionally redundant kinases [40]. In mammals, PKD and $\mathrm{Pkc} \delta$ act in combatting oxidative stress (overview in: $[34,58]$ ), conforming to a similar role for PKD in the fly [40]. Drak has an important role in epithelial tissue morphogenesis in Drosophila, in agreement with the involvement of PKD in the regulation of cytoskeletal dynamics $[59,60]$. Perhaps, Drak and PKD act in the regulation of eye to epithelial transformation as well, which is defective in $L$ mutants [25]. Finally, Sqa has a role in starvation-induced autophagy and the regulation of TOR signalling activity, linking PKD to growth control $[61,62]$. As long as the nature $L$ mutation remains unknown, however, we unfortunately 

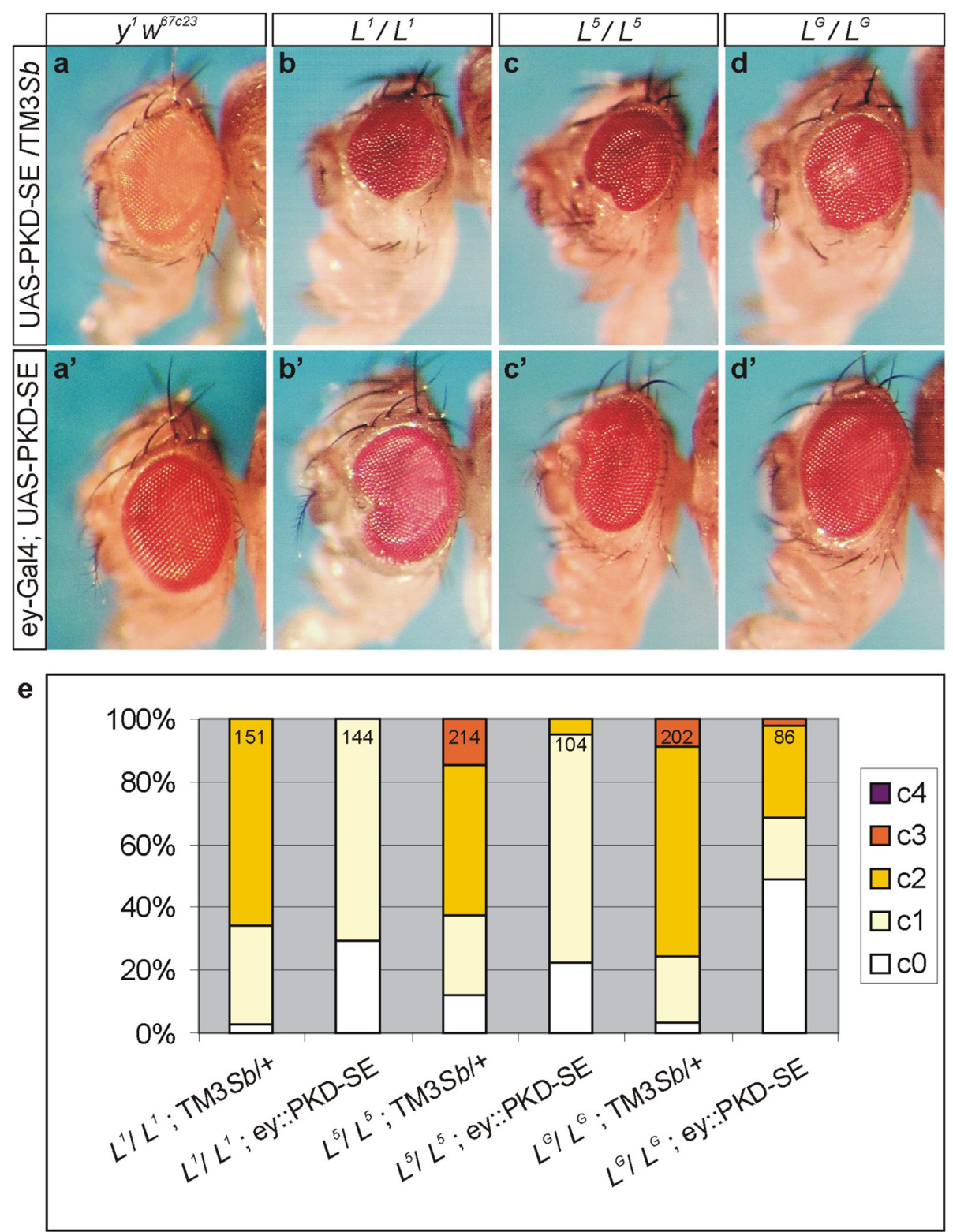

Fig. 5 Overexpression of PKD-SE rescues the small eye phenotype of several $L$ alleles. Typical examples of female heads are shown. (a-d) Upper row: Eye phenotype of the three homozygous Lobe alleles, $L^{1}(\mathbf{b}), L^{5}(\mathbf{c})$, and $L^{G}(\mathbf{d})$ in the background of UAS-PKD-SE, which does not affect the eye of the control $y^{7} w^{67 c 23}(\mathbf{a})$. (a'-d') Lower row: Overexpression of UAS-PKD-SE with ey-Gal4 (ey::PKD-SE) does not disturb outer eye morphology $\left(a^{\prime}\right)$, however, results in a rescue of the small eye of any tested $L$ allele $\left(b^{\prime}-d^{\prime}\right)$. (e) Quantification of eye phenotypes in the given genotype, using the following classification: $\mathrm{CO}$, wild type eyes; $\mathrm{c1}$, one eye is smaller; $\mathrm{C2}$, both eyes are smaller; $\mathrm{c} 3$, one eye is smaller and one is of pinhead size or absent; $\mathrm{c} 4$ both eyes are of pinhead size or absent. Genotypes are depicted below each column; (+) indicates third chromosome normal for PKD. Fractions of phenotypes are depicted; numbers of assessed females are indicated in each column. The animals were derived from a cross of $L^{*} / \mathrm{CyO}$; ey-Gal4/TM3Sb $\times L^{*} / \mathrm{CyO} ; \mathrm{UAS}-\mathrm{PKD}-\mathrm{SE} / \mathrm{TM} 3 \mathrm{~S} b$ at $18^{\circ} \mathrm{C}$

can only speculate as to the molecular basis of the $P K D-L$ interaction.

\section{Conclusion}

The Ser/Thr kinase PKD is required for the regulation of growth and cell survival during eye development, which is uncovered by the sensitized background of the $L$ mutation. Both, the fact that the PKD- $L$ interaction is not allele specific and that it is bidirectional, i.e. enhancement of $L$ in the absence of PKD and rescue of $L$ by overactivity of $P K D$, supports the specificity of the genetic interaction. Most likely, PKD-mediated protein phosphorylation is involved in underlying molecular processes causing the $L$ phenotype, i.e. in the regulation of growth, the epidermal transformation of eye tissue and apoptosis, respectively. The enigmatic nature of the $L$ 
mutation, however, only allows speculations as to the molecular basis of this interaction.

\section{Materials and methods}

The following fly stocks were used: Oregon $\mathrm{R}$ (OreR) and $y^{1} w^{67 c 23}$ (BL6599), $L^{1}$ (BL318), $L^{2}$ (BL319), $L^{5}$ (BL321), $L^{G}$ (BL322), PKD ${ }^{26}$ [40]; ey-Gal4 [42], UASPKD-WT, UAS-PKD-SE, UAS-PKD-kd [36]. Further information on fly strains is available at flybase.org. Flies were raised under non-crowded conditions on standard agar-corn-molasses food at $18{ }^{\circ} \mathrm{C}$ or $25^{\circ} \mathrm{C}$ as indicated. Crosses and combinations were performed with standard genetic techniques; analyses were performed on one to 5 days old flies. Presence of the $P K D^{26}$ allele in the recombinants was confirmed by PCR using the primer pair: P6 Cre-lox LP, 5' CCG GAC AGT GGA CTC ACA TA 3' and P8 white UP, 5' AAA AGT GCA GCG GAA ATA GTT A 3' [40]. Microphotographs of adult heads were taken with a Pixera ES120 digital camera (Optronics) coupled to a Leica M5 using the Pixera Viewfinder Version 2.0 software. Figures were assembled using Corel Photo Paint, Corel Draw, Exel, and BoxPlotR software. Statistical significance of probes was determined by ANOVA two-tailed test for multiple comparisons using Dunnet's approach with $p$-values: $p>0.05$ (not significant); $p<0.05 ; p<0.01 ; p<0.001$.

\section{Acknowledgements}

We are indebted to A. Cerny for the initial phenotypic analyses of the PKD ${ }^{26}$ and Lobe recombinants. We greatly acknowledge H. Gloc, I. Wech and E. Zettler for invaluable technical assistance.

\section{Authors' contribution}

$\mathrm{DM}, \mathrm{ACN}$ and $\mathrm{AP}$ conceived and designed the experiments and analyzed the data. AP wrote the manuscript, and all authors approved its final version.

\section{Funding}

The work was funded by the German science foundation with a grant MA 1328/8-1 to DM. The funder had no role in study design, data collection and analysis, decision to publish or preparation of the manuscript.

\section{Availability of data and materials}

The datasets supporting the conclusions of this article are included within the article.

\section{Ethics approval and consent to participate}

Not applicable.

\section{Consent for publication \\ Not applicable.}

\section{Competing interests}

The author declares that there are no competing financial, personal, or professional interests.

Received: 28 August 2019 Accepted: 10 December 2019 Published online: 19 December 2019

\section{References}

1. Shingleton AW. The regulation of organ size in Drosophila: physiology, plasticity, patterning and physical force. Organogenesis. 2010;6:76-87.

2. Kumar JP. My what big eyes you have: how the Drosophila retina grows. Dev Neurobiol. 2011;71(12):1133-52.
3. Kumar JP. The fly eye: through the looking glass. Dev Dyn. 2018;47(1):11123.

4. Gehring WJ, Ikeo K. Pax6 mastering eye morphogenesis and eye evolution. Trends Genet. 1999:15:371-7.

5. Gehring WJ. New perspectives on eye development and the evolution of eyes and photoreceptors. J Hered. 2005;96(3):171-84.

6. Hoge MA. Another gene in the fourth chromosome of Drosophila. Am Nat. 1915:49:47-9.

7. Fristrom D. Cellular degeneration in the production of some mutant phenotypes in Drosophila melanogaster. Mol Gen Genet. 1969;103:363-79.

8. Ransom R. The time of action of three mutations affecting Drosophila eye morphogenesis. J Embryol Exp Morpholog. 1979;53:225-35.

9. Kronhamn J, Frei E, Daube M, Jiao R, Shi Y, Noll M, Rasmuson-Lestander A. Headless flies produced by mutations in the paralogous Pax6 genes eyeless and twin of eyeless. Development. 2002;129(4):1015-26.

10. Zhu J, Palliyil S, Ran C, Kumar JP. Drosophila Pax6 promotes development of the entire eye-antennal disc, thereby ensuring proper adult head formation. Proc Natl Acad Sci U S A. 2017;114:5846-53.

11. Cho KO, Choi KW. Fringe is essential for mirror symmetry and morphogenesis in the Drosophila eye. Nature. 1998;396:272-6.

12. Dominguez $\mathrm{M}$, de Celis JF. A dorsal/ventral boundary established by Notch controls growth and polarity in the Drosophila eye. Nature. 1998;396:276-8.

13. Papayannopoulos V, Tomlinson A, Panin VM, Rauskolb C, Irvine K. Dorsalventral signaling in the Drosophila eye. Science. 1998;281:2031-4.

14. Cavodeassi F. Diez del corral R, Campuzano S, Dominguez M. compartments and organising boundaries in the Drosophila eye: the role of the homeodomain Iroquois proteins. Development. 1999:126(22):4933-42.

15. Singh A, Choi KW. Initial state of the Drosophila eye before dorsoventral specification is equivalent to ventral. Development. 2003;130(25):6351-60.

16. Lindsley D, Zimm GG. The genome of Drosophila melanogaster. San Diego CA: Academic Press; 1992.

17. Baker BS, Ridge KA. Sex and the single cell. I. on the action of the major loci affecting sex determination in Drosophila melanogaster. Genetics. 1980;94(2): 383-423.

18. Chern JJ, Choi KW. Lobe mediates Notch signaling to control domainspecific growth in the Drosophila eye disc. Development. 2002;129(17): 4005-13.

19. Medvedev NN. Genes and development of characters. I. the study of the growth of the imaginal discs of eyes of the wild type larvae and three mutants - Lobe ${ }^{c}$, glass ${ }^{2}$ and eyeless ${ }^{2}$ in Drosophila melanogaster. Z Indukt Abstamm Vererbungsl. 1935;70:55-72.

20. Steinberg AG. Studies on the development of the eye: evidence that the Lobe $^{2}$, Lobe $^{4}$, Lobe $^{5}$, and eyeless ${ }^{2}$ mutants of Drosophila melanogaster develop in a manner similar to Bar. Proc Natl Acad Sci U S A. 1944;30:5-13.

21. Zimm GG. An analysis of growth abnormalities associated with the eyemutant Lobe in Drosophila melanogaster. J Exp Zool. 1951;116:289-319.

22. Singh A, Chan J, Chern JJ, Choi KW. Genetic interaction of Lobe with its modifiers in dorsoventral patterning and growth of the Drosophila eye. Genetics. 2005:171:169-83.

23. Singh A, Shi X, Choi KW. Lobe and Serrate are required for cell survival during early eye development in Drosophila. Development. 2006;133(23): 4771-81.

24. Pichaud F, Casares F. homothorax and iroquois-C genes are required for the establishment of territories within the developing eye disc. Mech Dev. 2000; 96(1):15-25.

25. Singh A, Tare M, Kango-Singh M, Son WS, Cho KO, Choi KW. Opposing interactions between homothorax and Lobe define the ventral eye margin of Drosophila eye. Dev Biol. 2011;359(2):199-208.

26. Dunn LC. Coyne. The relationship between the effects of certain mutations on developmental rate and on adult characters. Biol. Zentbl. 1935;55:385-9.

27. Dunn LC, Mossige JC. The effects of the Minute mutations of Drosophila melanogaster on developmental rate. Hereditas. 1935;23:70-90.

28. Wang $\mathrm{YH}$, Huang ML. Reduction of Lobe leads to TORC1 hypoactivation that induces ectopic Jak/STAT signaling to impair Drosophila eye development. Mech Dev. 2009;126(10):781-90.

29. Pallares-Cartes C, Cakan-Akdogan G, Teleman AA. Tissue-specific coupling between insulin/IGF and TORC1 signaling via PRAS40 in Drosophila. Dev Cell. 2012;22(1):172-82.

30. Wullschleger S, Loewith R, Hall MN. TOR signaling in growth and metabolism. Cell. 2006;24:471-84. 
31. van Lint J, Rykx A, Maeda Y, Vantus T, Sturany S, Malhotra V, Vandenheede $J R$, Seufferlein T. Protein Kinase D: in intra-cellular traffic regulator on the move. Trends Cell Biol. 2002;12:193-200.

32. Rykx A, De Kimpe L, Mikhalap S, Vantus T, Seufferlein T, Vandenheede JR, Van Lint J. Protein Kinase D: a family affair. FEBS Lett. 2003;546:81-6.

33. Olayioye MA, Barišić S, Hausser A. Multi-level control of actin dynamics by protein Kinase D. Cell Signal. 2013;25(9):1739-47.

34. Cobbaut M, Van Lint J. Function and regulation of protein Kinase D in oxidative stress: a tale of isoforms. Oxidative Med Cell Longev. 2018;2018: 2138502. https://doi.org/10.1155/2018/2138502.

35. Maier D, Hausser A, Nagel AC, Link G, Kugler SJ, Wech I, Pfizenmaier K, Preiss A. Drosophila protein Kinase $D$ is broadly expressed and a fraction localizes to the Golgi compartment. Gene Expr Patterns. 2006;6(8):849-56.

36. Maier D, Nagel AC, Gloc H, Hausser A, Kugler SJ, Wech I, Preiss A. Protein Kinase $D$ regulates several aspects of development in Drosophila melanogaster. BMC Dev Biol. 2007;7:74.

37. Nagel AC, Schmid J, Auer JS, Preiss A, Maier D. Constitutively active protein Kinase $D$ acts as a negative regulator of slingshot-phosphatase in Drosophila. Hereditas. 2010;147(5):237-42.

38. Barišić S, Nagel AC, Franz-Wachtel M, Macek B, Preiss A, Link G, Maier D, Hausser A. Phosphorylation of Ser 402 impedes phosphatase activity of slingshot 1. EMBO Rep. 2011;12(6):527-33.

39. Ashe S, Malhotra V, Raghu P. Protein Kinase D regulates metabolism and growth by controlling secretion of insulin like peptide. Dev Biol. 2018;434(1): 175-85.

40. Maier D, Nagel AC, Kelp A, Preiss A. Protein Kinase D is dispensable for development and survival of Drosophila melanogaster. G3 (Bethesda). 2019; 9(8):2477-87.

41. Brand $\mathrm{AH}$, Perrimon N. Targeted gene expression as a means of altering cell fates and generating dominant phenotypes. Development. 1993;118(2):401-15.

42. Hazelett DJ, Bourois M, Walldorf U, Treisman JE. Decapentaplegic and wingless are regulated by eyes absent and eyegone and interact to direct the pattern of retinal differentiation in the eye disc. Development. 1998;125: 3741-51.

43. Ashe S, Yadav S. Maintenance of rhodopsin levels in Drosophila photoreceptor and phototransduction requires protein Kinase D. Fly (Austin). 2018;12(3-4):164-73.

44. Patterson JT, Muller HJ. Are 'progressive' mutations produced by X-rays? Genetics. 1930;15:495-577.

45. Halder G, Callaerts P, Flister S, Walldorf U, Kloter U, Gehring WJ. Eyeless initiates the expression of both sine oculis and eyes absent during Drosophila compound eye development. Development. 1998;125(12):2181-91.

46. Callaerts $P$, Leng $S$, Clements J, Benassayag C, Cribbs D, Kang YY, Walldorf U, Fischbach KF, Strauss R. Drosophila Pax-6/eyeless is essential for normal adult brain structure and function. J Neurobiol. 2001:46(2):73-88.

47. Clements J, Hens K, Merugu S, Dichtl B, de Couet HG, Callaerts P. Mutational analysis of the eyeless gene and phenotypic rescue reveal that an intact eyeless protein is necessary for normal eye and brain development in Drosophila. Dev Biol. 2009:334(2):503-12.

48. Noll M. Evolution and role of Pax genes. Curr Opin Genet Dev. 1993;3:595-605.

49. Zhang W, Zheng S, Storz P, Min W. Protein Kinase D specifically mediates apoptosis signal-regulating kinase 1-JNK signaling induced by $\mathrm{H}_{2} \mathrm{O}_{2}$ but not tumor necrosis factor. J Biol Chem. 2005:280:19036-44.

50. Vollmer J, Fried P, Sanchez-Aragon M, Lopes CS, Casares F, Iber D. A quantitative analysis of growth control in the Drosophila eye disc Development. 2016;143:1482-90.

51. Colombani J, Andersen DS, Leopold P. Secreted peptide Dilp8 coordinates Drosophila tissue growth with developmental timing. Science. 2012;336: 582-5.

52. Garelli A, Gontijo AM, Miguela V, Caparros E, Dominguez M. Imaginal discs secrete insulin-like peptide 8 to mediate plasticity of growth and maturation. Science. 2012;336:579-82.

53. Colombani J, Andersen DS, Boulan L, Boone E, Romero N, Virolle V, Texada M, Leopold P. Drosophila Lgr3 couples organ growth with maturation and ensures developmental stability. Curr Biol. 2015;25:2723-9.

54. Vallejo DM, Juarez-Carreno S, Bolivar J, Morante J, Dominguez M. A brain circuit that synchronizes growth and maturation revealed through Dilp8 binding to Lgr3. Science. 2015;350(6262):aac6767.

55. Boulan L, Andersen D, Colombani J, Boone E, Léopold P. Inter-Organ growth coordination is mediated by the Xrp1-Dilp8 axis in Drosophila. Dev Cell. 2019;49(5):811-818.e4.
56. Clements J, Hens K, Francis C, Schellens A, Callaerts P. Conserved role for the Drosophila Pax6 homolog eyeless in differentiation and function of insulin-producing neurons. Proc Natl Acad Sci U S A. 2008;105(42):16183-8.

57. Buhler K, Clements J, Winant M, Bolckmans L, Vulsteke V, Callaerts P. Growth control through regulation of insulin signalling by nutrition-activated steroid hormone in Drosophila. Development. 2018;145(21):dev165654.

58. Storz P. Mitochondrial ROS--radical detoxification, mediated by protein Kinase D. Trends Cell Biol. 2007;17(1):13-8.

59. Neubueser D, Hipfner DR. Overlapping roles of Drosophila Drak and rho kinases in epithelial tissue morphogenesis. Mol Biol Cell. 2010;21(16):2869-79.

60. Chougule AB, Hastert MC, Thomas JH. Drak is required for actomyosin organization during Drosophila cellularization. G3 (Bethesda). 2016;6(4):819-28.

61. Findlay $\mathrm{GL}$, Yan L, Procter J, Mieulet V, Lamb RF. A MAP 4 kinase related to Ste20 is a nutrient-sensitive regulator of mTOR signalling. Biochem J. 2007; 430(1):13-20.

62. Tang HW, Wang YB, Wang SL, Wu MH, Lin SY, Chen GC. Atg-1 mediated myosin II activation regulates autophagosome formation during starvation induced autophagy. EMBO J. 2011;30:636-51.

\section{Publisher's Note}

Springer Nature remains neutral with regard to jurisdictional claims in published maps and institutional affiliations.
Ready to submit your research? Choose BMC and benefit from:

- fast, convenient online submission

- thorough peer review by experienced researchers in your field

- rapid publication on acceptance

- support for research data, including large and complex data types

- gold Open Access which fosters wider collaboration and increased citations

- maximum visibility for your research: over $100 \mathrm{M}$ website views per year

At $\mathrm{BMC}$, research is always in progress.

Learn more biomedcentral.com/submissions 